\title{
Socioeconomic variations in the course of stroke: unequal health outcomes, equal care?
}

\section{G A M van den Bos, J P J M Smits, G P Westert, A van Straten}

J Epidemiol Community Health 2002;56:943-948

See end of article for authors' affiliations

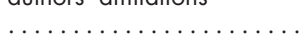

Correspondence to: Professor G A M van den Bos, National Institute of Public Health and the Environment, Department for Health Services

Research, PO Box 1, 3720

BA Bilthoven, Netherlands;

GAM.van.den.Bos@rivm.nl

Accepted for publication 28 February 2002

\begin{abstract}
Study objective: The aim of this paper is to quantify the socioeconomic gap in long term health outcomes after stroke and related health care utilisation, in order to evaluate whether those in need of care do actually receive appropriate levels of care.

Design: Stroke patients from the lower socioeconomic group were compared with stroke patients from the higher socioeconomic group with respect to sociodemographic and clinical characteristics, health outcomes, and related health care utilisation.

Setting: Patients were recruited from admissions to 23 randomly selected hospitals in the Netherlands. Patients: 465 patients were included who had had a stroke six months earlier and were followed up three years and five years after stroke.

Main results: The observed odds ratios suggest that patients from the lower socioeconomic group experienced more disabilities up to three years after stroke and more handicaps up to five years after stroke. After adjusting for health care needs there were no significant associations between socioeconomic status and health care utilisation. The observed figures, however, suggest that a lower socioeconomic status tended to increase admission to nursing homes and to decrease receiving care in non-institutional settings.

Conclusions: Overall, inequalities in long term health outcomes were observed but solid indications for large inequalities in health care utilisation were not found. More investments in coordinated stroke services are needed to alleviate the unfavourable health situation of disadvantaged groups and to ensure that health care services respond appropriately to the health care needs of different socioeconomic groups.
\end{abstract}

S troke has a major impact on health and health care. Mortality rates are high, and many survivors have impaired functional health outcomes and rely on a wide variety of healthcare services for a prolonged period of time. In the Netherlands, the annual incidence is estimated to be $185 / 100000$ and the point prevalence 545/100 000. ${ }^{1}$ Stroke is the third cause of death and a major cause of disabilities. More than $3 \%$ of the Dutch annual healthcare budget is spent on stroke patients. ${ }^{2}$ There are reasons to expect that some subgroups, especially stroke patients from the lower socioeconomic groups, are experiencing a substantially higher disease burden. The risk of mortality is considerably higher in stroke patients from lower socioeconomic groups. ${ }^{3-6}$ Socioeconomic status affects the incidence and prevalence of stroke and other manifestations of cardiovascular disease. ${ }^{78}$ Though clear associations are not found for all risk factors for cardiovascular diseases, research findings indicate that lower socioeconomic groups have a worse risk profile for cardiovascular diseases..$^{8-12}$ While these studies have shown that people from lower socioeconomic groups are at a greater risk for stroke morbidity and stroke mortality, less progress has been made in describing the impact of socioeconomic status on health outcomes in the long term and on related health care utilisation. From patient and population studies it is known that stroke has a large impact on general health and ensuing health care needs. ${ }^{13-24}$ Research findings lend support to the hypothesis that stroke patients in lower socioeconomic groups are exposed to a double disadvantage or are subjected to what has been called a double suffering, ${ }^{25}$ not only in that there is a relatively high prevalence of stroke among them, but also in that the impact of stroke upon them is greater. In addition, we expect that stroke patients from the lower socioeconomic groups-who are less healthy-use more health care. When applying the principle of equity in health care-one of the basic quality indicators of the Dutch health care system - the variations in health care utilisation between lower and higher socioeconomic groups should disappear when health related needs are taken into account. ${ }^{26-28}$ The principle of equity presupposes that utilisation of care is predominantly determined by clinical and health characteristics; inequity is present if care is explained by factors enabling or impeding use of health care, such as socioeconomic status.

It is not clear whether socioeconomic differentials in health and health care utilisation decline or expand during the course of stroke. ${ }^{29}$ Socioeconomic differences in long term health outcomes and related health care utilisation may decrease because survivors from lower socioeconomic groups represent a relatively healthy subgroup; the more fragile patients die in an earlier phase. Alternatively, the socioeconomic gap in health and health care may diverge. ${ }^{30}$ Because of cumulative advantages during life span, the positive effect of social status on health and health care is expected to increase in the course of time, producing more inequality in health between lower and higher status groups. The aim of this article is to quantify the socioeconomic gap in (a) long term health outcomes after stroke, and (b) related health care utilisation after controlling for long term health outcomes, in order to evaluate whether those in need of care receive appropriate levels of care.

\section{METHODS}

\section{Study group and data collection}

The study group consisted of 465 patients who had had a stroke six months earlier. These patients originated from a cohort of 760 stroke patients who participated in a multicentre study on quality of care in the Netherlands. ${ }^{162831}$ All 760 patients were admitted within one week after stroke onset to 
23 randomly selected Dutch hospitals. This patient cohort was followed up from hospital admission up to five years after stroke. Clinical data on stroke (stroke severity, stroke type, and previous strokes) and demographic data (age and gender) were abstracted from the patients' medical and nursing hospital records. Six months, three years, and five years after stroke, patients were interviewed at home. If patients were not communicative because of cognitive, speech or language disorders, patient data were collected in a proxy interview, usually with the partner.

Because no data were available on the socioeconomic status of patients who died within six months after stroke, our analyses necessarily were confined to the six months survivors. Of the 502 patients who were still alive after six months, 17 patients refused to participate in the interview and for 20 patients socioeconomic status-that is, educational level-was unknown. For some of the remaining 465 patients, information on one or more of the other variables was missing. Information on the pattern of missing values of the variables used in this study can be found in table 1 .

\section{Measures}

To quantify the impact of socioeconomic status on long term health outcomes of stroke and related health care utilisation, data were collected on (a) sociodemographic characteristics, (b) clinical characteristics, (c) health outcomes, and (d) health care utilisation.

\section{Sociodemographic characteristics}

The data concerned were age, gender, and socioeconomic status. Socioeconomic status was assessed on the basis of educational level into two groups: (a) primary education or lower level secondary education, and (b) intermediate secondary or higher education. No additional data were available on income or occupation.

\section{Clinical characteristics}

Severity of index stroke, defined in terms of level of consciousness, was assessed with the Glasgow Coma Scale. ${ }^{32}$ Because of possible aphasia, the verbal component was deleted. A patient was considered to be alert when the score on the Eyes or Motor component was maximal. Type of index stroke was subdivided into infratentorial strokes and supratentorial strokes (lacunar infarctions, (sub)cortical infarctions, or intracerebral haemorrhages). A recurrent stroke was defined present if a stroke was reported in the period before or after the index stroke.

\section{Long term health outcomes}

Follow up data were collected on disability, handicap, and perceived health. Disability in activities of daily living (ADL) was assessed with the Barthel Index, ranging from 0 to 20 (ADL independent if score $=20) .{ }^{33}$ Handicap was measured with the modified Rankin Scale, ranging from 0 to 5 (severe handicap if score $\geqslant 3) .{ }^{34}{ }^{35}$ Health perception was assessed with a single item question (How would you rate your present health?), dichotomised into good self rated health (feeling healthy) and poor self rated health (feeling unhealthy).

\section{Long term utilisation of health care}

Utilisation of health care was assessed for a wide range of health services. For brevity, we aggregated health care services into broader categories according to location and function of care: (1) non-institutional care; (2) rehabilitation centre; (3) institutional care (nursing home, residential home). Noninstitutional care was subdivided into (la) therapy (physical therapy, occupational therapy, and speech therapy); (lb) (I)ADLcare (home help, nursing care and day care); ( lc) psychosocial support ( social care, mental care, and support from fellow patients); (ld) in addition, patients were categorised as "high" care users if they used three or more separate health care services.

\section{Statistical analysis}

Firstly, we analysed univariate relations between socioeconomic status, health outcomes, and health care utilisation among six months survivors. To identify socioeconomic differences $\chi^{2}$ tests were performed. Additionally, the independent impact of socioeconomic status on long term health outcomes and health care utilisation was analysed by multivariate logistic regression analyses using data six months, three years, and five years after stroke. In the regression analyses of long term health outcomes, we adjusted for demographic and clinical variables. The variables selected were age, gender, stroke type, stroke severity, and recurrent stroke. In a previous study we found that these characteristics were major prognostic factors on health outcomes. ${ }^{24}$ To estimate the effect of socioeconomic status on health care utilisation, long term health outcomes were added to the regression model, besides demographic and clinical variables. The effect sizes were expressed as odds ratios (ORs) (calculated as the antilogarithm of the regression coefficients of the logistic regression model) with 95\% confidence intervals. The problem of the possible selective effect of socioeconomic status on mortality was tackled by repeating the analyses including only the survivors of five years after stroke. The cumulative mortality was 34\% at six months, $51 \%$ at three years, and $62 \%$ at five years. Estimates of the magnitude of socioeconomic differences were based on the range measure comparing the lower educational group with the higher educational group. ${ }^{36}$ The higher educational group was taken as reference group. All statistical analyses were performed with the statistical program SPSS 10.0.

\section{RESULTS}

\section{Profile of the patients in the study group}

The mean age of the 465 patients was 71 years (range 20-95 years), 214 patients (46\%) were women and 308 patients $(66 \%)$ belonged to the lower socioeconomic group. Table 1 describes the characteristics of the patients in the study group by socioeconomic status. Stroke patients from the lower socioeconomic group were older in comparison with stroke patients from the higher socioeconomic group. The majority of women were classified in the lower socioeconomic group.

Ten per cent of the patients were affected with a severe stroke. About $15 \%$ of the patients suffered from an infratentorial stroke and $85 \%$ from a supratentorial stroke (lacunar infarction 19\%, (sub)cortical infarction 53\%, intracerebral haemorrhage 13\%). Approximately 19\% of the patients reported a recurrent stroke. The observed figures suggest that patients from the lower socioeconomic group tended to have more frequently a severe stroke and a (sub)cortical infarction, and less frequently a lacunar infarction.

A substantial number of patients were experiencing unfavourable health outcomes. Disability was observed in 61\% of the patients, 59\% reported handicaps, and $43 \%$ felt unhealthy. The health outcomes in terms of disability and handicap were significantly worse in stroke patients from the lower socioeconomic group. No significant socioeconomic gradient was found for health perception.

About $20 \%$ of the patients lived in institutions, $5 \%$ stayed in rehabilitation centres, and $75 \%$ were still living in the community. Of all non-institutionalised patients, $32 \%$ did not use any care at all. The other patients received various types of care: therapy (38\%), (I)ADL care (45\%) and psychosocial support $(32 \%)$. Almost a quarter of these patients used three or more services. The health care utilisation profile of the lower socioeconomic group was characterised by significantly more admissions to nursing homes, and consequently fewer opportunities to stay at home. Non-institutionalised patients from 
Table 1 Demographic, clinical, and health characteristics, and health care utilisation among survivors six months after stroke, by socioeconomic status (SES)

\begin{tabular}{|c|c|c|c|}
\hline & Low SES (\%) & High SES (\%) & Total (\%) \\
\hline \multicolumn{4}{|l|}{ Demographic characteristics } \\
\hline Age $>70.53$ (mean age) & $198 / 308(64.3)$ & $68 / 157(43.3)$ & $266 / 465(57.2)^{*}$ \\
\hline Gender (female) & $173 / 308(56.2)$ & $41 / 157(26.1)$ & $214 / 465(46.0)^{*}$ \\
\hline \multicolumn{4}{|l|}{ Clinical characteristics } \\
\hline Severe stroke & $30 / 301(10.0)$ & $13 / 154(8.4)$ & $43 / 455(9.5)$ \\
\hline \multicolumn{4}{|l|}{ Type of index stroke } \\
\hline Infratentorial stroke & $44 / 292(15.1)$ & $20 / 148(13.5)$ & $64 / 440(14.5)$ \\
\hline Lacunar infarction & $51 / 292(17.5)$ & $35 / 148(23.6)$ & $86 / 440(19.5)$ \\
\hline (Sub)cortical infarction & $161 / 292(55.1)$ & $73 / 148(49.3)$ & $234 / 440(53.2)$ \\
\hline Haemorrhage & $36 / 292(12.3)$ & $20 / 148(13.5)$ & $56 / 440(12.7)$ \\
\hline Recurrent stroke & $57 / 308$ (18.5) & $31 / 157(19.7)$ & $88 / 465$ (18.9) \\
\hline \multicolumn{4}{|l|}{ Health outcomes } \\
\hline Disability in ADL & $211 / 306(69.0)$ & $67 / 147(45.6)$ & $278 / 453(61.4)^{*}$ \\
\hline Handicap & $201 / 306(65.7)$ & $65 / 146(44.5)$ & $266 / 452(58.8)^{*}$ \\
\hline Poor self rated health & $91 / 225(40.4)$ & $60 / 124(48.4)$ & $151 / 349(43.3)$ \\
\hline \multicolumn{4}{|l|}{ Health care utilisation } \\
\hline Non-institutionalised patients & $217 / 307(70.7)$ & $123 / 146(84.2)$ & $340 / 453(75.1)^{*}$ \\
\hline No care & $58 / 215(27.0)$ & $50 / 122(41.0)$ & $108 / 337(32.0)^{*}$ * \\
\hline Therapy & $83 / 216(38.4)$ & $45 / 122(36.9)$ & $128 / 338(37.9)$ \\
\hline (I) ADL-care & $110 / 215(51.2)$ & $43 / 122(35.2)$ & $153 / 337(45.4)^{*}$ \\
\hline Psychosocial support & $64 / 213(30.0)$ & $42 / 122(34.4)$ & $106 / 335(31.6)$ \\
\hline High care & $49 / 217(22.6)$ & $30 / 123(24.4)$ & $79 / 340(23.2)$ \\
\hline Rehabilitation centre & $15 / 307(4.9)$ & $6 / 146(4.1)$ & $21 / 453(4.6)$ \\
\hline Institutional care & $75 / 307(24.4)$ & $17 / 146(11.6)$ & $92 / 453(20.3)^{*}$ \\
\hline
\end{tabular}

Table 2 Logistic regression models to demonstrate the association between long term health outcomes and socioeconomic status among survivors six months $(n=443)$, three years $(n=309)$, and five years $(n=229)$ after stroke, adjusted for demographic and clinical characteristics; odds ratios (95\% confidence intervals), reference group are patients from the higher socioeconomic group

\begin{tabular}{llll}
\hline & Six months & Three years & Five years \\
\hline Disability in ADL & $1.67(1.03$ to 2.72$)$ & $1.39(0.79$ to 2.45$)$ & $1.09(0.56$ to 2.13$)$ \\
Handicap & $1.75(1.08$ to 2.83$)$ & $1.66(0.93$ to 2.95$)$ & $1.67(0.81$ to 3.45$)$ \\
Poor self rated health & $0.66(0.41$ to 1.07$)$ & $1.21(0.68$ to 2.15$)$ & $0.82(0.43$ to 1.58$)$ \\
\hline
\end{tabular}

Table 3 Logistic regression models to demonstrate the association between long term health outcomes and socioeconomic status among five years survivors $(n=229)$ after stroke, adjusted for demographic and clinical characteristics; odds ratios $195 \%$ confidence intervals), reference group are patients from the higher socioeconomic group

\begin{tabular}{llll}
\hline & Six months & Three years & Five years \\
\hline Disability in ADL & $1.58(0.81$ to 3.09$)$ & $1.34(0.69$ to 2.58$)$ & $1.09(0.56$ to 2.13$)$ \\
Handicap & $1.68(0.85$ to 3.35$)$ & $1.71(0.87$ to 3.36$)$ & $1.67(0.81$ to 3.45$)$ \\
Poor self rated health & $0.70(0.35$ to 1.40$)$ & $1.32(0.68$ to 2.57$)$ & $0.82(0.43$ to 1.58$)$ \\
\hline
\end{tabular}

the lower socioeconomic group received significantly more community care, particularly (I)ADL care.

\section{Long term health outcomes}

In tables 2 and 3 the data on the long term health outcomes are presented by socioeconomic status, after adjusting for demographic and clinical characteristics. The observed ORs suggest that patients from the lower socioeconomic group reported more disabilities up to three years after stroke (ORs respectively $1.67,1.39$ ), and more handicaps up to five years after stroke (ORs respectively 1.75, 1.66, 1.67) (table 2). The socioeconomic differences for disability were smaller than for handicap, and disappeared a few years after stroke. No clear socioeconomic pattern was observed for health perception.
Table 3 demonstrates that the diminishing effect of socioeconomic status on disability was not attributable to selective mortality.

\section{Long term utilisation of health care}

Table 4 shows the adjusted utilisation figures for the health services under study among the stroke survivors six months, three years, and five years after stroke. In contrast with the univariate analyses, the multivariate analyses did not reveal significant socioeconomic differences in health care utilisation. The observed ORs suggest that patients from the lower socioeconomic group were more likely to be admitted to nursing homes in the long term (ORs respectively 1.01, 1.39, 1.52) and consequently were less likely to live in the community. 
Table 4 Logistic regression models to demonstrate the association between long term health care utilisation and socioeconomic status among survivors six months $(n=438)$, three years $(n=306)$, and five years $(n=228)$ after stroke, adjusted for demographic, clinical, and long term health characteristics; odds ratios $195 \%$ confidence intervals), reference group are patients from the higher socioeconomic group

\begin{tabular}{llll}
\hline & Six months & Three years & Five years \\
\hline Non-institutionalised patients & $1.07(0.52$ to 2.19$)$ & $0.72(0.26$ to 2.02$)$ & $0.66(0.17$ to 2.49 \\
No care & $0.78(0.44$ to 1.38$)$ & $1.14(0.60$ to 2.18$)$ & $1.18(0.53$ to 2.63$)$ \\
Therapy & $0.92(0.52$ to 1.61$)$ & $0.63(0.29$ to 1.37$)$ & $0.56(0.22$ to 1.38$)$ \\
(I)ADL-care & $1.05(0.60$ to 1.85$)$ & $1.06(0.53$ to 2.12$)$ & $0.72(0.29$ to 1.77$)$ \\
Psychosocial support & $0.74(0.43$ to 1.28$)$ & $0.68(0.31$ to 1.51$)$ & $1.03(0.41$ to 2.57$)$ \\
High care & $0.71(0.38$ to 1.33$)$ & $0.89(0.30$ to 2.64$)$ & $0.80(0.20$ to 3.20$)$ \\
Rehabilitation centre & $0.88(0.29$ to 2.70$)$ & - & - \\
Institutional care & $1.01(0.44$ to 2.32$)$ & $1.39(0.50$ to 3.92$)$ & $1.52(0.40$ to 5.78$)$ \\
\hline & & & \\
\hline
\end{tabular}

Table 5 Logistic regression models to demonstrate the association between long term health care utilisation and socioeconomic status among five years survivors $(n=228)$ after stroke, adjusted for demographic, clinical, and long term health characteristics; odds ratios ( $95 \%$ confidence intervals), reference group are patients from the higher socioeconomic group

\begin{tabular}{llll}
\hline & Six months & Three years & Five years \\
\hline Non-institutionalised patients & $2.17(0.57$ to 8.24$)$ & $1.35(0.28$ to 6.59$)$ & $0.66(0.17$ to 2.49 \\
No care & $0.74(0.35$ to 1.55$)$ & $1.40(0.65$ to 3.01$)$ & $1.18(0.53$ to 2.63$)$ \\
Therapy & $0.87(0.40$ to 1.88$)$ & $0.66(0.26$ to 1.67$)$ & $0.56(0.22$ to 1.38$)$ \\
(I)ADL-care & $1.02(0.47$ to 2.19$)$ & $0.83(0.35$ to 1.96$)$ & $0.72(0.29$ to 1.77$)$ \\
Psychosocial support & $0.68(0.33$ to 1.42$)$ & $0.45(0.18$ to 1.13$)$ & $1.03(0.41$ to 2.57$)$ \\
High care & $0.61(0.27$ to 1.40$)$ & $0.95(0.26$ to 3.49$)$ & $0.80(0.20$ to 3.20$)$ \\
Rehabilitation centre & $0.75(0.15$ to 3.84$)$ & - & - \\
Institutional care & $0.28(0.04$ to 2.08$)$ & $0.74(0.15$ to 3.62$)$ & $1.52(0.40$ to 5.78$)$ \\
\hline
\end{tabular}

Compared with table 4 , table 5 showed much higher ORs ratios for non-institutionalised patients at six months and three years after stroke, and consequently much lower ORs for institutional care. This suggests that stroke patients with higher socioeconomic status are less likely to die in nursing homes.

\section{DISCUSSION}

In this study we quantified socioeconomic differences in long term health outcomes and related health care utilisation among stroke patients.

Previous studies have demonstrated that patients with a lacunar infarction reported better overall functioning than patients with a supratentorial stroke, and that patients with (sub)cortical infraction were more probably in a poor health. ${ }^{164}$ Our data suggest that patients with a lower socioeconomic status were experiencing a stroke with a worse clinical profile.

In addition, patients with lower socioeconomic status seemed to have significantly worse health outcomes in terms of disability and handicap six months after stroke. The higher disease burden in the lower socioeconomic group lends support to the phenomenon of "double suffering" - the lower socioeconomic groups have not only a higher incidence of stroke and a worse risk profile, but they are also more vulnerable to functional impairments. In the long run we observed declining socioeconomic differences for disability but not for handicap. Our study did not find firm indications for a widening or decreasing socioeconomic gap in health outcomes in the course of time.

While a socioeconomic gradient was observed for the more objective health indicators, such as disability and handicap, no clear gradient was found for health perceptions. It is well

\section{Key points}

- Stroke patients in lower socioeconomic groups have worse long term health outcomes.

- There are no solid indications for large inequalities in health care utilisation among stroke patients.

- Stroke patients in lower socioeconomic groups are more likely to be admitted to nursing homes and are less likely to live in the community.

- Community based health care programmes should be developed further to support independent living, particularly where socioeconomic disadvantages are at stake.

- More investments in coordinated stroke services are needed to alleviate the unfavourable health situation of disadvantaged groups.

known that self ratings of health are not only based on physical or functional aspects of health but also on attitudes, expectations and adaptation to worsening health outcomes. ${ }^{37} 38$ This suggests that subjective health perceptions are less useful in monitoring health outcomes.

Differential utilisation of health care services by stroke patients may contribute to the explanation of socioeconomic differences in health outcomes during the course of stroke. Our study did not produce solid evidence for this explanation. After all, our data demonstrated a positive relation between health related needs and utilisation of health care services. After adjusting for demographic, clinical, and health characteristics, there were no significant associations. However, in some respects socioeconomic inequalities in health care utilisation cannot be ruled out. Our data suggest that a lower 
socioeconomic status tended to increase admission to nursing homes, and to decrease receiving care in non-institutional settings.

The strength of our study is the integration of medical data and patients' data. We could rely on professional diagnostics, and could consider a range of clinical characteristics, such as severity and type of stroke. Most studies in the field of socioeconomic inequalities in health(care) are based on self reported illness, relying on the information given by patients. ${ }^{173940}$ We performed a prospective study with a long follow up period up to five years after stroke, which enables us to include a broad spectrum of health care services: non-institutional as well as institutional care, and rehabilitation as well as supportive care.

A few remarks should be made in interpreting the results of our study. Firstly, generalisation of our findings may be affected by selection of hospitalised patients. In the Netherlands approximately $30 \%$ of all stroke patients are not admitted to a hospital. ${ }^{41}$ These are patients either with a very poor prognosis or with a rapidly reversible stroke. However, the mortality risk we found in our stroke population was remarkable similar to several community based studies..$^{24}$ There are no specific data available to assess potential selection bias attributable to socioeconomic status. As the adjusted overall pattern of hospital admission does not show a clear socioeconomic gradient, ${ }^{39}{ }^{40}$ we do not expect a substantial distortion in the association between socioeconomic status, health outcomes, and health care utilisation. Secondly, the use of education as indicator of socioeconomic status is not without discussion. On the one hand, education rather than income and occupation is considered to be a more stable indicator of lifelong socioeconomic status for the elderly and a more consistent predictor of health. ${ }^{30}$ On the other hand, education may cause a lack of differentiation at the bottom of the distribution, because today's older population has less academic qualifications, particularly women..$^{42-44}$ This may result in an underestimation of socioeconomic differences. Nevertheless, we found no conclusive evidence for this bias when repeating the analyses separately for men. Thirdly, the low numbers may have induced a lack of power in the analyses. Consequently, we may not have been able to identify relevant differences. Fourthly, we only examined whether or not patients used health care services. Assessments of utilisation in a dichotomous way, however, are an unspecific indicator of the quality of health care. We know little about the frequency and intensity of care delivered. Fifthly, by examining the health care users, we excluded a group of patients who perceived unmet demands for health care. A previous study has shown that unmet demands were reported by a substantial number of patients $(31 \%$ of the non-institutionalised patients six months after stroke), and that these demands were not related to socioeconomic level. ${ }^{28}$ This finding suggests that the actual health care demands exceed the actual rate of health care utilisation, but that there is no selection bias according to socioeconomic status. Lastly, we only focused on the use of health care in relation to patient characteristics. We know that patient characteristics alone do not explain variations in health care, and that structure and process indicators have a substantial influence on long term health outcomes. ${ }^{45}{ }^{46}$ Stroke units, for example, seem to improve considerably patient outcomes in the long term, and the observed benefits are not restricted to any particular subgroup of patients or model of stroke unit care. ${ }^{47-49}$

To summarise, our research hypothesis "Socioeconomic variations in the course of stroke: unequal health outcomes, equal care" is not falsified by our data. Our study has shown that in addition to well known socioeconomic inequalities in mortality and morbidity, there are inequalities in long term health outcomes. Overall, we found no solid indications for inequalities in health care utilisation. However, some inequitable access to health care cannot be ruled out as to whether patients receive non-institutional care or are admitted to nursing homes. As patients prefer to stay home as long as possible, more efforts are needed to develop community based health care programmes and to support independent living, particularly where socioeconomic disadvantages are at stake. More investments in coordinated stroke services are needed to alleviate the unfavourable health situation of disadvantaged groups and to ensure that health care services respond appropriately to the health care needs of different socioeconomic groups.

\section{ACKNOWLEDGMENTS}

The research reported is part of the Research on Stroke Amsterdam (ROSA), a multicentre study on quality of care conducted by the Academic Medical Centre/University of Amsterdam. The departments involved are Social Medicine (Professor dr G A M van den Bos), Neurology (Professor dr M Limburg) and Clinical Epidemiology and Biostatistics (Professor dr R J de Haan). We thank Dr H C Boshuizen for her methodological advice and comments.

\section{Authors' affiliations}

G A M van den Bos, National Institute of Public Health and the Environment, Department for Health Services Research and Academic Medical Centre, Department for Social Medicine, Netherlands

J P J M Smits, G P Westert, National Institute of Public Health and the Environment, Department for Health Services Research, Netherlands A van Straten, Netherlands Institute of Mental Health and Addiction, Netherlands

Funding: ROSA was supported by grants from the Dutch Organisation for Scientific Research, the Netherlands Heart Foundation and the Dutch Fund for Investigative Medicine of the Health Insurance Council.

Conflicts of interest: none.

\section{REFERENCES}

1 Ruwaard D, Kramers PGN, eds. Public health status and forecasts 1997: Health, prevention and health care in the Netherlands until 2015. Maarssen: Elsevier/De Tijdstroom, 1997.

2 Evers SMAA, Engel GL, Ament AJHA. Cost of stroke in the Netherlands from a societal perspective. Stroke 1997;28:1375-81.

3 Siegel PZ, Deeb LC, Wolfe LE, et al. Stroke mortality and its socioeconomic, racial and behavioral correlates in Florida. Public Health Rep 1993; 108:454-8.

4 Bennett S. Socioeconomic inequalities in coronary heart disease and stroke mortality among Australian men, 1979-1993. Int J Epidemiol 1996:25:266-75

5 Kunst $\mathbf{A E}$, del Rios M, Groenhof F, et al. Socioeconomic inequalities in stroke mortality among middle-aged men: an international overview. European Union Working Group on Socioeconomic Inequalities in Health. Stroke 1998;29:2285-91.

6 Maheswaran R, Elliot P, Strachan DP. Socioeconomic deprivation, ethnicity and stroke mortality in Greater London and south east England. J Epidemiol Community Health 1997;51:127-31.

7 Reeder BA, Liu L, Horlick L. Sociodemographic variation in the prevalence of cardiovascular disease. Can J Cardiol 1996;12:271-7.

8 Van Rossum CTM, van de Mheen H, Breteler MMB, et al. Socioeconomic differences in stroke among Dutch elderly women? The Rotterdam Study. Stroke 1999;30:357-62.

9 Lynch JW, Kaplan GA, Cohen RD, et al. Do cardiovascular risk factors explain the relation between socioeconomic status, risk of all-cause mortality, cardiovascular mortality, and acute myocardial infarction? Am J Epidemiol 1996;144:934-42.

10 Davey Smith G, Ben-Shlomo Y. Geographical and social class differentials in stroke mortality - the influence of early-life factors: comment on papers by Maheswaran and colleagues. J Epidemiol Community Health 1997:51:134-7.

11 Davey Smith G, Hart C, Blane D, et al. Adverse socioeconomic conditions in childhood and cause specific adult mortality: prospective observational study. BM 1998;316:1631-5.

12 Lynch JW, Everson SA, Kaplan GA, et al. Does low socioeconomic status potentiate the effects of heightened cardiovascular responses to stress on the progression of carotid atherosclerosis? Am J Public Health stress on the progresi
1998;88:389-94.

13 Schuling J, Greidanus J, Meyboom-de Jong B. Measuring functional status of stroke patients with the Sickness Impact Profile. Disabil Rehabil 1993;15:19-23.

14 Guccione AA, Felson DT, Anderson JJ, et al. The effects of specific medical conditions on the functional limitations of elders in the Framingham Study. Am J Public Health 1994;84:351-8.

15 Boult C, Kane RL, Louis TA, et al. Chronic conditions that lead to functional limitation in the elderly. J Gerontol Med Sci 1994;49:M28-36. 
16 De Haan R, Limburg M, van der Meulen J, et al. Quality of life after stroke: impact of stroke type and lesion location. Stroke 1995;26:402-8.

17 Van den Bos GAM. The burden of chronic diseases in terms of disability, use of health care and healthy life expectancies. Eur J Public Health 1995:5:29-34.

18 Tuomilehto J, Nuottimaki T, Salmi K, et al. Psychosocial and health status in stroke survivors after 14 years. Stroke 1995;26:971-5.

19 Verbrugge LM, Patrick DL. Seven chronic conditions: their impact on US adults' activity levels and use of medical services. Am J Public health 1995:85:173-82.

20 Hochstenbach J, Donders R, Mulder T, et al. Long-term outcome after stroke: a disability-orientated approach. Int J Rehabil Res 1996; 19:189-200

21 Picavet HSJ, van den Bos GAM. The contribution of six chronic conditions to the total burden of mobility disability in the Dutch population. Am J Public Health 1997;87: 1680-2.

22 Bonita R, Solomon N, Broad JB. Prevalence of stroke and stroke-related disability. Stroke 1997;28:1898-902.

23 Wilkinson PR, Wolfe CDA, Warburton FG, et al. A long-term follow-up of stroke patients. Stroke 1997;28:507-12.

24 Van Straten A, Reitsma JB, Limburg $M$, et al. Impact of stroke type on survival and functional health. Cerebrovasc Dis 2001;12:27-33.

25 Blank N, Diderichsen F. Social inequalities in the experience of illness in Sweden: a "double suffering". Scand J Soc Med 1996;24:81-9.

26 De Haan $\mathbf{R}$, Limburg $M$, van der Meulen J, et al. Use of health care services after stroke. Quality in Health Care 1993;2:222-7.

27 Andersen RM. Revisiting the behavioral model and access to medical care: does it matter? J Health and Soc Behav 1995;36:1-10.

28 Scholte op Reimer WJM, de Haan RJ, Rijnders PT, et al. Unmet care demands after stroke: deficits in health care? Quality in Health Care 1999;8:30-5.

29 Breeze E, Sloggett A, Fletcher AE. Socioeconomic status and transitions in status in old age in relation to limiting long-term illness measured at the 1991 Census. Eur J Public Health 1999;9:265-70.

30 Ross CE, WU CL. Education, age and the cumulative advantage in health. J Health Soc Behav 1996;37: 104-20.

31 Van Straten A, van der Meulen JHP, van den Bos GAM, et al. Length of hospital stay and discharge delays in stroke patients. Stroke 1997;28:137-40.

32 Teasdale G, Jenett B. Assessment of coma and impaired consciousness. Lancet $1974 ;$ ii: 81.

33 Wade DT, Collin C. The Barthel ADL index: a standard measure of physical disability? Int Disabil Stud 1988;10:64-7.
34 Bamford JM, Sandercock PAG, Warlow CP, et al. Interobserver agreement for the assessment of handicap in stroke patients. [Letter] Stroke 1989;20:828

35 De Haan R, Limburg $M$, Bossuyt $P$, et al. The clinical meaning of Rankin 'handicap' grades. Stroke 1995;26:2027-30.

36 Wagstaff A, Paci P, Van Doorslaer E. On the measurement of inequalities in health. Soc Sci Med 1991;33:545-57.

37 Hoeymans N, Feskens EJM, van den Bos GAM, et al. Age time, and cohort effects on functional status and self-rated health in elderly men. Am $J$ Publ Health 1997;87: 1620-5.

38 Hoeymans N, Feskens EJM, Kromhout D, et al. Ageing and the relationship between functional status and self-rated health. Soc Sci Med 1997;45: 1527-36.

39 Van den Bos GAM van der Maas PJ. Social inequalities in the basic components of health expectancy: chronic morbidity, disability and mortality. In: Robine J-M, Mathers CD, Bone MR et al, eds. Calculation of health expectancies: harmonization, concensus achieved and future perspectives. Montrouge: Colloque INSERM/ John Libbey Eurotext, 1993;226: 193-201.

40 Van der Meer JBW, van den Bos J, Mackenbach JP. Socio-economic differences in the utilization of health services in a Dutch population: the contribution of health status. Health Policy 1996;37:1-18.

41 Bots M, Looman SJ, Koudstaal PJ, et al. Prevalence of stroke in the general population. The Rotterdam Study. Stroke 1996;27: 1499-501.

42 Stronks K, van de Mheen H, van den Bos J, et al. Smaller socioeconomic inequalities in health among women: the role of employment status. Int J Epidemiol 1995;24:559-68.

43 Sacker A, Firth D, Fitzpatrick $R$, et al. Comparing health inequality in men and women: prospective study of mortality 1986-96. BM 2000;320:1303-7.

44 Holt G, Grundy E, Sacker A, et al. Comparing health inequality in men and women. [Ltter]. BM 2000;321:961.

45 McKevitt CJ, Beech R, Pound P, et al. Putting stroke outcomes into context. Eur J Public Health 2000:10:120-6.

46 Van den Bos GAM, Triemstra AHM. Quality of life as an instrument for need assessment and outcome assessment of health care in chronic patients. Quality in Health Care 1999;8:247-52.

47 Langhorne $\mathbf{P}$, Williams BO, Gilchrist W, et al. Do stroke units save lives? Lancet 1993;342:395-8.

48 Stroke Unit Trialists' Collaboration. Collaborative systematic review of the randomised trials of organised inpatient (stroke unit) care after stroke. Stroke Unit Trialists' Collaboration. BM 1997;314:1151-9.

49 Indredavik B, Bakke F, Slordahl SA, et al. Stroke unit treatment improves long-term quality of life: a randomized controlled trial. Stroke 1998;29:895-9. 